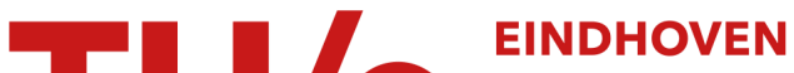 UNIVERSITY OF TECHNOLOGY
}

\section{Signatures of resonance superfluidity in a quantum Fermi gas}

\section{Citation for published version (APA):}

Chiofalo, M. L., Kokkelmans, S. J. J. M. F., Milstein, J. N., \& Holland, M. J. (2002). Signatures of resonance superfluidity in a quantum Fermi gas. Physical Review Letters, 88(9), 090402-1/4. [090402]. https://doi.org/10.1103/PhysRevLett.88.090402

DOI:

10.1103/PhysRevLett.88.090402

Document status and date:

Published: 01/01/2002

\section{Document Version:}

Publisher's PDF, also known as Version of Record (includes final page, issue and volume numbers)

\section{Please check the document version of this publication:}

- A submitted manuscript is the version of the article upon submission and before peer-review. There can be important differences between the submitted version and the official published version of record. People interested in the research are advised to contact the author for the final version of the publication, or visit the $\mathrm{DOI}$ to the publisher's website.

- The final author version and the galley proof are versions of the publication after peer review.

- The final published version features the final layout of the paper including the volume, issue and page numbers.

Link to publication

\section{General rights}

Copyright and moral rights for the publications made accessible in the public portal are retained by the authors and/or other copyright owners and it is a condition of accessing publications that users recognise and abide by the legal requirements associated with these rights.

- Users may download and print one copy of any publication from the public portal for the purpose of private study or research.

- You may not further distribute the material or use it for any profit-making activity or commercial gain

- You may freely distribute the URL identifying the publication in the public portal.

If the publication is distributed under the terms of Article 25fa of the Dutch Copyright Act, indicated by the "Taverne" license above, please follow below link for the End User Agreement:

www.tue.nl/taverne

Take down policy

If you believe that this document breaches copyright please contact us at:

openaccess@tue.nl

providing details and we will investigate your claim. 


\title{
Signatures of Resonance Superfluidity in a Quantum Fermi Gas
}

\author{
M.L. Chiofalo,* S. J. J. M. F. Kokkelmans, J.N. Milstein, and M. J. Holland \\ JILA, University of Colorado and National Institute of Standards and Technology, \\ Boulder, Colorado 80309-0440
}

(Received 5 October 2001; published 12 February 2002)

\begin{abstract}
We predict a direct and observable signature of the superfluid phase in a quantum Fermi gas, in a temperature regime already accessible in current experiments. We apply the theory of resonance superfluidity to a gas confined in a harmonic potential and demonstrate that a significant increase in density will be observed in the vicinity of the trap center.
\end{abstract}

DOI: 10.1103/PhysRevLett.88.090402

PACS numbers: 03.75.Fi, 67.60.-g, 74.20.-Z

Following the successful realization of Bose-Einstein condensation (BEC) in confined vapors [1], it is natural to consider possibilities for observing the analogous superfluid phase transition in a dilute Fermi gas. Quantum degeneracy has already been demonstrated in a twocomponent Fermi gas of ${ }^{40} \mathrm{~K}$ atoms [2], although the spin states utilized were not suitable for exhibiting superfluidity. The lowest temperatures achieved to date in this system are around $0.2 T_{F}$ - limited by Pauli blocking as well as a number of technical considerations [3]. In other experiments, the rethermalization of fermion atoms by elastic collisions with a bath of ultracold bosons is exploited; realized by mixtures of ${ }^{6} \mathrm{Li}$ and ${ }^{7} \mathrm{Li}$ at Rice [4], at ENS [5], and at Duke [6], and more recently by mixtures of ${ }^{40} \mathrm{~K}$ atoms and ${ }^{87} \mathrm{Rb}$ atoms at JILA [7].

In order to observe a superfluid phase transition at critical temperatures as high as $0.2 T_{F}$, the existence of a strong coupling mechanism which could lead to a significant amount of Cooper pairing is necessary. Several theoretical papers have presented models to investigate this regime, essentially based on application of the Bardeen-Cooper-Schrieffer (BCS) [8] theory of superconductivity. These approaches consider dilute Fermi vapors in which the two-body scattering processes are characterized by a large negative scattering length $a$ [9]. Under such conditions the relevant length scale - the spatial extent of the Cooper pair - may become comparable to the average interparticle spacing. This places the system in a crossover region from the BCS superfluidity of momentumcorrelated fermion pairs to the BEC of tightly bound composite bosons. In this crossover regime, fluctuations play a crucial role [10] and must be addressed.

Eventually, as the coupling is increased, it becomes necessary to construct a theory in which explicit treatment of the composite bosonic states is made. Such an approach was proposed in the context of high-temperature superconductivity [11] and is based on an effective manybody Hamiltonian, in which quasibound pairs are explicitly treated as resonance states embedded in the continuum of the Fermi sea. Such resonances are ubiquitous in atomic physics, where, for example, a Feshbach resonance [12] can be utilized to tune a quasibound state through thresh- old, providing an explicit microscopic basis for a theory of resonance superfluidity $[13,14]$.

A convincing method for detecting the superfluidity will be required. Various approaches have been proposed; including measurements of the pair distribution [15], experiments involving the breakup of the Cooper pairs [16], measurements of the moment of inertia [17], and probes of collective excitations $[18,19]$. In this Letter, we show that a more straightforward and direct experimental signature of the transition to the superfluid phase is provided by the density characteristics in an inhomogeneous system. We demonstrate that in a harmonic trap, the superfluid state is manifest as the appearance of a bulge in the central atomic density. To this aim we derive a theory of resonance superfluidity including the description of external confinement.

Although our results will have general applicability, for the purpose of illustration, we consider a typical system of $N=5 \times 10^{5}$ atoms in an isotropic harmonic trap with angular frequency $\omega=2 \pi \times 100 \mathrm{~s}^{-1}$. This gives a Fermi energy of $E_{F}=(3 N)^{1 / 3} \hbar \omega$. We consider a ${ }^{40} \mathrm{~K}$ Feshbach resonance, illustrated in Fig. 1, for $s$-wave scattering of atoms in the lowest two hyperfine spin states which we denote by $\sigma \in\{\uparrow, \downarrow\}$. We begin by considering the general structure of the theory for the homogeneous system where the fermions are represented by the wave-number- $k$ dependent annihilation operators $a_{k \sigma}$ and the composite boson field by $b_{\boldsymbol{k}}$. The Hamiltonian is

$$
\begin{aligned}
H= & \sum_{\boldsymbol{k} \sigma} \epsilon_{k} a_{\boldsymbol{k} \sigma}^{\dagger} a_{\boldsymbol{k} \sigma}+\nu \sum_{\boldsymbol{k}} b_{\boldsymbol{k}}^{\dagger} b_{\boldsymbol{k}} \\
& +U \sum_{\boldsymbol{q} \boldsymbol{k} \boldsymbol{k}^{\prime}} a_{\boldsymbol{q} / 2+\boldsymbol{k} \uparrow}^{\dagger} a_{\boldsymbol{q} / 2-\boldsymbol{k} \downarrow}^{\dagger} a_{\boldsymbol{q} / 2-\boldsymbol{k}^{\prime} \downarrow} a_{\boldsymbol{q} / 2+\boldsymbol{k}^{\prime} \uparrow} \\
& +\left(g \sum_{\boldsymbol{k} \boldsymbol{q}} b_{\boldsymbol{q}}^{\dagger} a_{\boldsymbol{q} / 2-\boldsymbol{k} \downarrow} a_{\boldsymbol{q} / 2+\boldsymbol{k} \uparrow}+\text { H.c. }\right),
\end{aligned}
$$

where H.c. denotes the Hermitian conjugate. The free dispersion relation for the fermions is $\epsilon_{k}$, and $\nu$ denotes the detuning of the boson resonance state from the zero edge of the collision continuum. The collisional interactions include both background fermion scattering and interconversion between composite bosons and fermion pairs. It is implicit in treating $U$ and $g$ as constants that the theory 


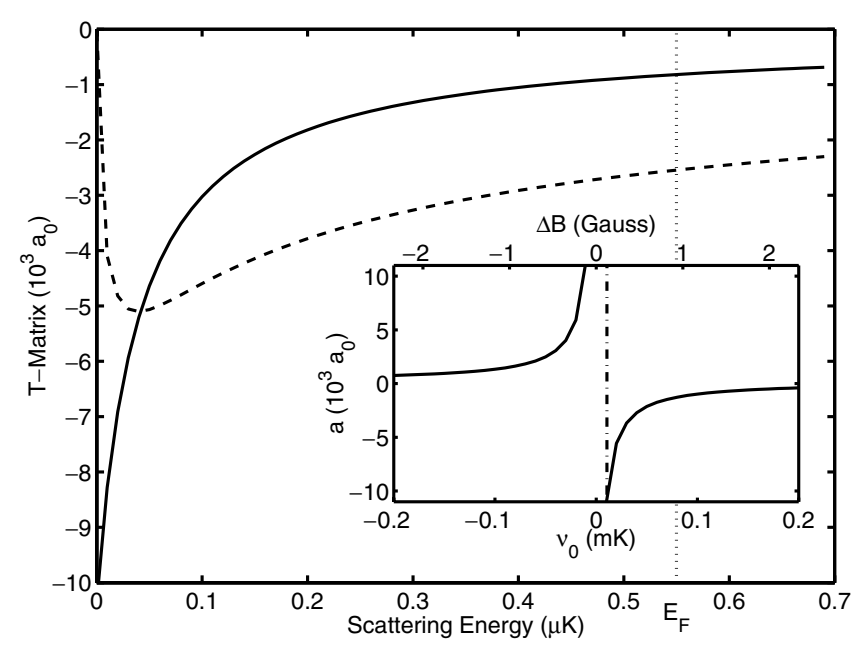

FIG. 1. Real (solid line) and imaginary (dashed line) components of the $T$ matrix for collisions of the lowest two spin states of ${ }^{40} \mathrm{~K}$ at a detuning of $20 E_{F}$, shown in length dimensions, i.e., $T_{k} /\left(4 \pi \hbar^{2} / m\right)$. The scattering length is the intercept at zero scattering energy which for this case is approximately $-10000 a_{0}$, where $a_{0}$ is the Bohr radius. The large variation in the $T$ matrix over the relevant energy range indicates that a quantum field theory developed from this microscopic basis will in general need to account for physics beyond the scattering length approximation. The inset shows the scattering length as a function of detuning, with $20 E_{F}$ detuning indicated by the dash-dotted line. This curve obeys the following form: $a=a_{\mathrm{bg}}\left(1-\kappa / \nu_{0}\right)$, where $a_{\mathrm{bg}}=176 a_{0}$ and $\kappa=0.657 \mathrm{mK}$ [20]. The quasipotentials to be renormalized are then $U_{0}=4 \pi \hbar^{2} a_{\mathrm{bg}} / m$ and $g_{0}=\sqrt{\kappa U_{0}}$.

will be renormalized and thereby contain no ultraviolet divergences in the calculation of observable quantities [21]. This procedure involves ascribing a cutoff value $K$ as the upper limit of all momentum summations and renormalizing the Hamiltonian constants in terms of $K$ and the parameters for the Feshbach resonance as given in Fig. 1. Defining $\alpha=m K /\left(2 \pi^{2} \hbar^{2}\right)$ and a dimensionless factor $\Gamma=\left(1-\alpha U_{0}\right)^{-1}$, the renormalization is executed by the following relations $U=\Gamma U_{0}, g=\Gamma g_{0}$, and $\nu=\nu_{0}+\alpha g g_{0}$. All results presented here have been shown to be independent of $K$.

From this Hamiltonian, we construct the dynamical Hartree-Fock-Bogoliubov equations for both the bosonic and fermionic mean fields. These equations involve the mean fields corresponding to the spin density $n=\sum_{k}\left\langle a_{k \sigma}^{\dagger} a_{k \sigma}\right\rangle$ (taken to be identical for both spins), the pairing field $p=\sum_{k}\left\langle a_{-k} \downarrow a_{k} \uparrow\right.$, and the condensed boson field $\phi_{m}=\left\langle b_{\boldsymbol{k}=0}\right\rangle$. The single particle density matrix

$$
G_{i j}=\left\langle A_{j}^{\dagger} A_{i}\right\rangle, \quad A=\left(\begin{array}{c}
a_{k \uparrow} \\
a_{k \downarrow} \\
a_{-k \uparrow}^{\dagger} \\
a_{-k \downarrow}^{\dagger}
\end{array}\right)
$$

evolves according to the Bogoliubov self-energy $\Sigma$,

$$
i \hbar \frac{d \mathcal{G}}{d t}=[\Sigma, G]
$$

The self-energy has Hermitian structure

$$
\Sigma=\left(\begin{array}{cccc}
U_{k} & 0 & 0 & \Delta \\
0 & U_{k} & -\Delta & 0 \\
0 & -\Delta^{*} & -U_{k} & 0 \\
\Delta^{*} & 0 & 0 & -U_{k}
\end{array}\right)
$$

where the single particle energy is $U_{k}=\epsilon_{k}-\mu+T_{k} n$, the gap is $\Delta=U p+g \phi_{m}$, and $\mu$ is the chemical potential. Here, without cost, we have upgraded the mean-field contribution to the single particle energy (which would otherwise be given by $U n$ ) to the full ladder sum, $T_{k} n$, where $T_{k}$ is the two-body $T$ matrix. This expression for the mean-field contribution to the particle energy is an approximation which is accurate for dilute Fermi gases where the quantum Fermi pressure limits achievable densities. The dynamical equations are closed by the evolution equation for the boson mode:

$$
i \hbar \frac{d \phi_{m}}{d t}=\nu \phi_{m}+g p
$$

The self-energy $\Sigma$ is diagonalized locally at each $\boldsymbol{k}$ by the Bogoliubov transformation generating quasiparticles with energy spectrum $E_{k}=\sqrt{U_{k}^{2}+|\Delta|^{2}}$. In equilibrium, the quasiparticle states are occupied according to the FermiDirac distribution $n_{k}=\left\{\exp \left[\left(E_{k}-\mu\right) / k_{b} T\right]+1\right\}^{-1}$. The corresponding maximum entropy solution for the molecule amplitude is found by $i \hbar d \phi_{m} / d t=\mu_{m} \phi_{m}$ where $\mu_{m}=2 \mu$, so that Eq. (5) implies $\phi_{m}=g p /$ $\left(\mu_{m}-\nu\right)$. The mean fields can then be determined by integration of the equilibrium single particle density matrix elements, given by

$$
\begin{aligned}
& n=\frac{1}{(2 \pi)^{2}} \int_{0}^{K} d k\left[\left(2 n_{k}-1\right) \cos 2 \theta_{k}+1\right], \\
& p=\frac{1}{(2 \pi)^{2}} \int_{0}^{K} d k\left(2 n_{k}-1\right) \sin 2 \theta_{k},
\end{aligned}
$$

where $\tan 2 \theta_{k}=|\Delta| / U_{k}$ is the Bogoliubov transformation angle. Since $\theta_{k}$ depends on $n$ and $p$, these equations require self-consistent solutions [22].

So far this theory has been presented for a homogeneous system, while we are interested in a gas of $N$ atoms confined in an external trapping potential $V(\boldsymbol{r})$. However, a full quantum mechanical treatment of the trapping states is not required. For instance, in our case, with a temperature of $T=0.2 T_{F}$, the harmonic oscillator level spacing is smaller than both the Fermi and thermal energies. Under these conditions, we may incorporate the effect of the trap through a semiclassical local density approximation [23]. This involves replacing the chemical potential by a local one $\mu(\boldsymbol{r})=\mu-V(\boldsymbol{r})$ and determining the thermodynamic solution at each point in space as for the homogeneous system.

In general, the validity of the semiclassical approximation requires a slow variation in the occupation of the discrete quantum levels as a function of energy. Remarkably, in both bosonic and fermionic gases, this condition 
can often be satisfied even at very low temperatures, because of strong correlations in a BEC due to repulsive interactions and because of exchange effects in a quantum Fermi gas. In both cases the zero-temperature semiclassical approximation for dilute gases is usually referred to as the Thomas-Fermi approximation.

We evaluate the thermodynamic quantities at given $T$ and $N$ in three steps: (i) For given $\mu$, we determine the local chemical potential $\mu(\boldsymbol{r})=\mu-V(\boldsymbol{r})$ and use this value to find the self-consistent solution for the density $n(\boldsymbol{r})$ and pairing field $p(\boldsymbol{r})$ at each point in space, according to the solution of Eqs. (6); (ii) we modify the global chemical potential $\mu$ until the density integral is the desired atom number, i.e., $N=\int d^{3} r n(\boldsymbol{r})$; (iii) we use the resulting solution for $\mu$ to calculate observable quantities, such as the density, gap, compressibility, and so forth.

The resulting solution for the density distribution is illustrated in Fig. 2. A striking signature of the resonance superfluidity is evident in the predicted density profile which has a notable bulge in the region of the center of the trap. Experimentally, this signature appears to be directly accessible. A typical approach would be to fit the expected density profile for a quantum degenerate gas with no superfluid phase to the wings of the distribution (outside the dotted lines shown in Fig. 2). Then the excess density observed at the trap center can be recorded. Figure 3 illustrates the emergence of the superfluid as the temperature is decreased. Qualitatively this situation is reminiscent of the central condensate peak observed for a Bose-Einstein condensed gas in a harmonic potential, although the connection appears to be somewhat serendipitous.

We explain the observed behavior by considering the compressibility of the normal and superfluid gases.

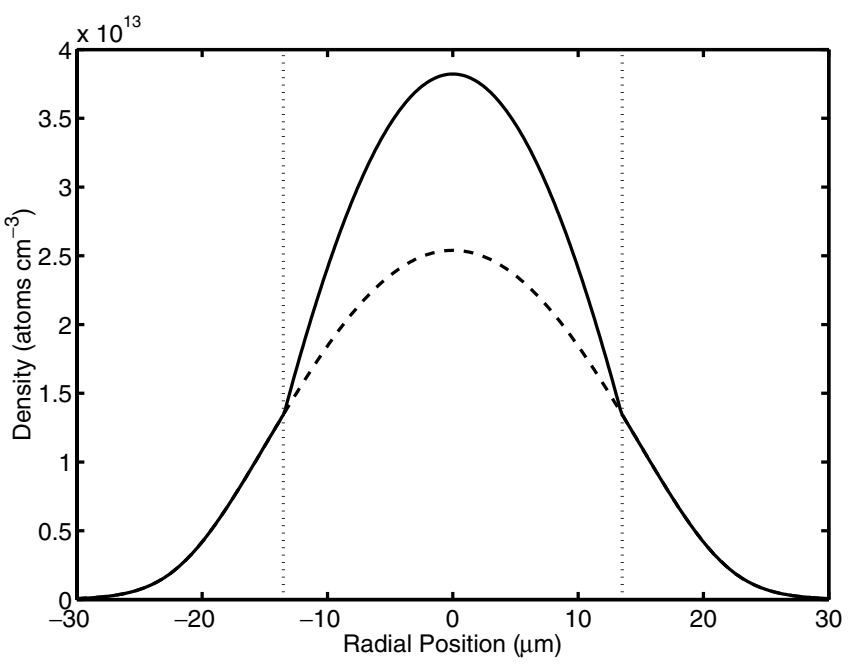

FIG. 2. Density profile at temperature $T=0.2 T_{F}$ and detuning $\nu_{0}=20 E_{F}$ showing accumulation of atoms at the trap center (solid line). We compare with the profile resulting from the same $\mu$ (but therefore a different total number of atoms) by artificially setting the pairing field $p$ to zero so that no superfluid is present (dashed line).
Thermodynamically, the isothermal compressibility $C$ is defined as $C^{-1}=n(\partial P / \partial n)_{T}$, where $P$ is the pressure, and is shown in Fig. 4. The compressibility is positive everywhere, indicating that, in spite of the large attractive interactions, the Fermi pressure makes the configuration mechanically stable. A significant feature is the discontinuous behavior at the radius from the trap center at which the superfluid changes from a zero to a nonzero value. This discontinuity is a manifestation of a second-order phase transition occurring in space. The discontinuity is a consequence of the local density approximation and cannot occur in a finite system. However, a rapid change in the compressibility is expected. In principle this could be probed by studies of shock waves generated by the abrupt jump in the speed of sound as a density fluctuation passes through this region.

In conclusion, we demonstrated that there exists a direct signature of superfluidity in trapped fermion gases. The onset of superfluidity leads to a density bulge in the center of the trap which can be detected by direct absorption imaging. The critical conditions for superfluidity are satisfied initially in the trap center, and the region of nonzero pairing field spreads out from the center as the temperature is lowered further. The increase in the density profile in the superfluid region is caused by a jump in the compressibility. Direct measures of this behavior are possible by the study of the propagation of sound waves. We have applied our method here to ${ }^{40} \mathrm{~K}$, but a similar approach is easy to derive for other interesting atoms, including, in particular, ${ }^{6} \mathrm{Li}$ which is the other fermionic alkali currently being investigated experimentally.

Support is acknowledged for M.H. and S. K. from the U.S. Department of Energy, Office of Basic Energy Sciences via the Chemical Sciences, Geosciences and Biosciences Division, and for M. C. from the National Science

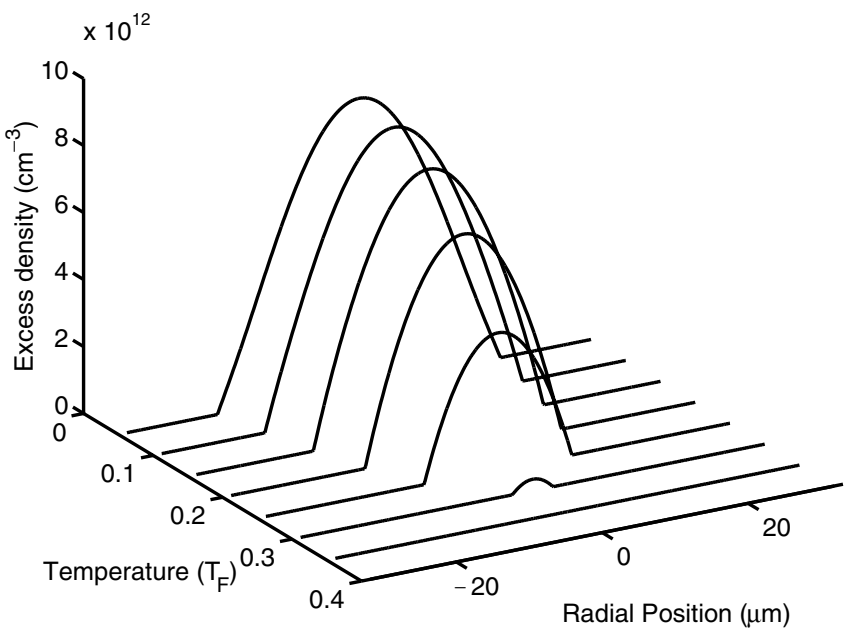

FIG. 3. Emergence of the coherent superfluid for $\nu_{0}=20 E_{F}$. The superfluid occupies an increasing volume as the temperature is reduced. Shown is the excess density (difference between the dashed and solid lines in Fig. 2) at each temperature. 


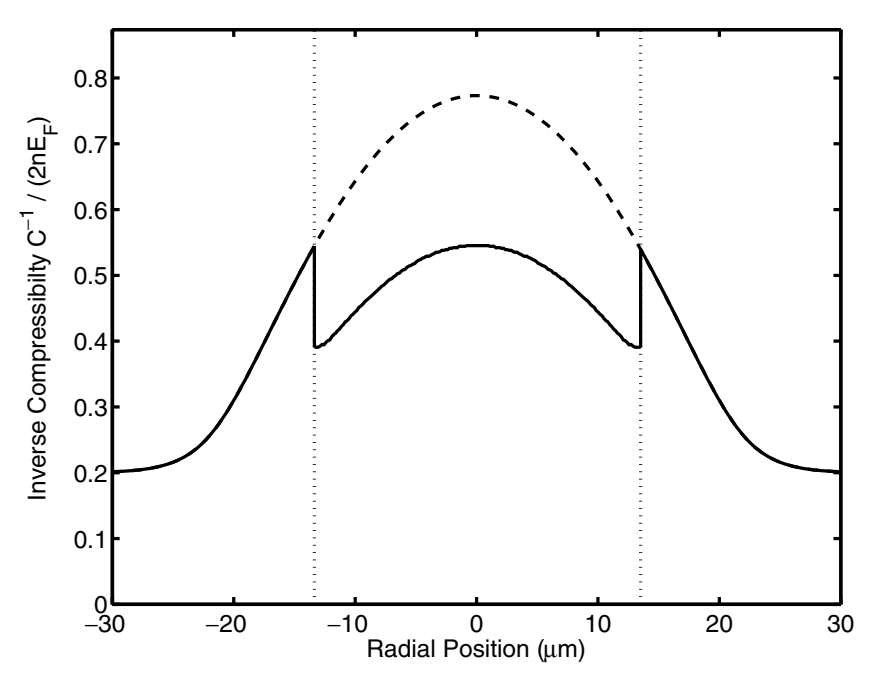

FIG. 4. Inverse isothermal compressibility $C^{-1}$ in units of the Fermi energy (solid line). Here $\nu_{0}=20 E_{F}$ and $T=0.2 T_{F}$ (as can be seen from the limiting behavior at the large radial position). A discontinuity appears at the radius at which the superfluid emerges (dotted line). We compare this solution to that corresponding to zero pairing field and no superfluid phase transition (dashed line).

Foundation and SNS, Pisa (Italy). We are grateful for the hospitality of the Aspen Center for Physics where this work was partially carried out. M.C. acknowledges the marvelous hospitality of JILA and thanks Ester Piegari for useful discussions.

*Permanent address: INFM and Classe di Scienze, Scuola Normale Superiore of Pisa, Pisa, Italy.

[1] M.H. Anderson, J.R. Ensher, M.R. Matthews, C.E. Wieman, and E. A. Cornell, Science 269, 198 (1995); K. B. Davis, M.-O. Mewes, M.R. Andrews, N. J. van Druten, D. S. Durfee, D. M. Kurn, and W. Ketterle, Phys. Rev. Lett. 75, 3969 (1995); C. C. Bradley, C. A. Sackett, J. J. Tollett, and R. G. Hulet, Phys. Rev. Lett. 75, 1687 (1995); 79, 1170(E) (1997).

[2] B. DeMarco and D. S. Jin, Science 285, 1703 (1999).

[3] M. J. Holland, B. DeMarco, and D. S. Jin, Phys. Rev. A 61, 053610 (2000); B. DeMarco, S. B. Papp, and D. S. Jin, Phys. Rev. Lett. 86, 5409 (2001).

[4] A. G. Truscott, K. E. Strecker, W. I. McAlexander, G. B. Partridge, and R. G. Hulet, Science 291, 2570 (2001).

[5] F. Schreck, L. Khaykovich, K. L. Corwin, G. Ferrari, T. Bourdel, J. Cubizolles, and C. Salomon, Phys. Rev. Lett. 87, 080403 (2001).

[6] S.R. Granade, M.E. Gehm, K. M. O’Hara, and J.E. Thomas, e-print cond-mat/0111344.

[7] J. Goldwin, S. B. Papp, B. DeMarco, and D. S. Jin, e-print cond-mat/0108287.
[8] J. Bardeen, L. N. Cooper, and J. R. Schrieffer, Phys. Rev. 108, 1175 (1957); J. R. Schrieffer, Theory of Superconductivity (Perseus Books, Reading, MA, 1999).

[9] H. T. C. Stoof, M. Houbiers, C. A. Sackett, and R. G. Hulet, Phys. Rev. Lett. 76, 10 (1996); M. Houbiers and H. T. C. Stoof, Phys. Rev. A 59, 1556 (1999); R. Combescot, Phys. Rev. Lett. 83, 3766 (1999); T. Papenbrock and G. F. Bertsch, Phys. Rev. C 59, 2052 (1999); G. Bruun et al., Eur. Phys. J. D 7, 433-439 (1999); H. Heiselberg, C. J. Pethick, H. Smith, and L. Viverit, Phys. Rev. Lett. 85, 2418 (2000).

[10] A. G. Leggett, J. Phys. (Paris), Colloq. 7, C7-19 (1980); P. Nozières and S. Schmitt-Rink, J. Low Temp. Phys. 59, 195 (1985); for a review, see M. Randeria, in Bose-Einstein Condensation, edited by A. Griffin, D. W. Snoke, and S. Stringari (Cambridge University Press, Cambridge, 1995).

[11] J. Ranninger and S. Robaszkiewicz, Physica (Amsterdam) 53B, 468 (1985); R. Friedberg and T. D. Lee, Phys. Rev. B 40, 6745 (1989); for a review, see V. Cataudella et al., in Models and Phenomenology for Conventional and High-Temperature Superconductivity, Proceedings of the International School of Physics "Enrico Fermi," edited by G. Iadonisi, J. R. Schrieffer, and M.L. Chiofalo (IOS Press, Amsterdam, 1998); J. Ranninger and J.-M. Robin, ibid.

[12] H. Feshbach, Ann. Phys. (N.Y.) 5, 357 (1958); E. Tiesinga, B. J. Verhaar, and H. T. C. Stoof, Phys. Rev. A 47, 4114 (1993); S. Inouye, M. R. Andrews, J. Stenger, H.-J. Miesner, D. M. Stamper-Kurn, and W. Ketterle, Nature (London) 392, 151 (1998).

[13] M. Holland, S. J. J. M. F. Kokkelmans, R. Walser, and M. L. Chiofalo, Phys. Rev. Lett. 87, 120406 (2001).

[14] E. Timmermans, K. Furuya, P. W. Milonni, and A. K. Kerman, Phys. Lett. A 285, 228 (2001).

[15] W. Zhang, C. A. Sackett, and R. G. Hulet, Phys. Rev. A 60, 504 (1999); J. Ruostekoski, ibid. 61, 033605 (2000); F. Weig and W. Zwerger, Europhys. Lett. 49, 282 (2000).

[16] P. Törma and P. Zoller, Phys. Rev. Lett. 85, 487 (2000).

[17] M. Farine, P. Schuck, and X. Viñas, Phys. Rev. A 62, 013608 (2000).

[18] A. Minguzzi, G. Ferrari, and Y. Castin, e-print condmat/0103591.

[19] M. A. Baranov and D.S. Petrov, Phys. Rev. A 62, 041601(R) (2000); A. Minguzzi and M.P. Tosi, Phys. Rev. A 63, 023609 (2001).

[20] John L. Bohn, Phys. Rev. A 61, 053409 (2000).

[21] S. J. J. M. F. Kokkelmans, J. Milstein, M. Chiofalo, R. Walser, and M. Holland, e-print cond-mat/0112283.

[22] The $p=0$ solution should be discarded when the superfluid state is present since it then corresponds to an unstable point on the free energy surface.

[23] V. Bagnato, D.E. Pritchard, and D. Kleppner, Phys. Rev. A 35, 4534 (1987); A. Minguzzi, S. Conti, and M. P. Tosi, J. Phys. Condens. Matter 9, L33 (1997); K. Mølmer, Phys. Rev. Lett. 80, 1804 (1998). 